\title{
Defining current facial fracture patterns in a quaternary institution following high-velocity blunt trauma
}

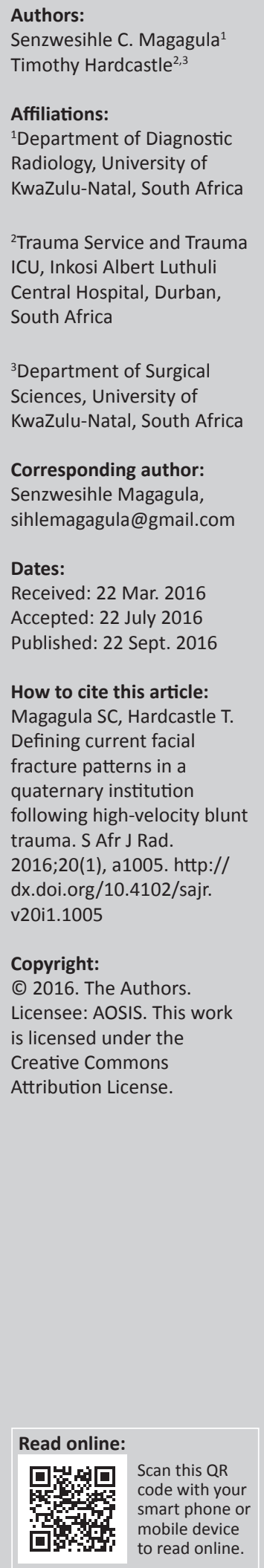

Background: In the early 20th century, René Le Fort studied facial fractures resulting from blunt trauma and devised a classification system still in common use today. This classification, however, was based on low-velocity trauma. In modern practice, in a quaternary-level referral hospital, patients are often admitted following high-velocity injuries that mostly result from motor vehicle collisions.

Objectives: A retrospective study to define facial bone fractures occurring subsequent to highvelocity trauma.

Method: A retrospective study comprising the review of CT scans of 52 patients with highvelocity facial fractures was performed between April 2007 and March 2013. Injuries were classified using the Le Fort classification system. Deviations from the true Le Fort types, which are often depicted in the literature as occurring bilaterally and symmetrically, were documented; these included unilaterality, occurrence of several Le Fort fractures on one side of the face, occurrence of several Le Fort fractures on different levels and on different sides of the face, and occurrence of other fractures in addition to Le Fort fractures.

Results: Of the 52 cases, 12 (23\%) had Le Fort injuries, with true Le Fort fractures occurring in only 1, and 11 deviating from the classic description. Nine patients had Le Fort fractures and additional fractures. Mandibular and zygomatic bone fractures were found to be common associations with Le Fort injuries, occurring in 58\% and 33\% of the cases respectively.

Conclusion: Fractures occurring in modern practice often deviate from the traditional Le Fort classification. Precise recognition of these deviations and recognition of additional associated fractures is pivotal in their management, assisting the surgeon in determining the treatment plan, such as the surgical approach and the order in which to fix the various fractured components.

\section{Introduction}

Around the turn of the 19th into the 20th centuries, René Le Fort, a renowned French surgeon, conducted a series of experiments using human cadavers to study facial bone fractures resulting from blunt trauma. Various forces were applied to inflict these injuries, some of which are controversial. The impacts included hitting the midface of a whole or decapitated cadaver with a baseball bat, and hitting the midface region onto a granite tabletop. He then cut the body segments open and studied the fractures they had sustained. Additionally, he boiled the skulls to strip off the flesh and expose the underlying fractures. He concluded that midface fractures occurred through 'lines' of inherent weakness in the facial skeletal structure, producing defined injury patterns. ${ }^{1}$ The fracture patterns were predictable and reproducible, depending on the site of impact on the midface. He then formulated a classification system still extensively used today: the Le Fort classification. Common to these fractures is involvement of the pterygoid plates, resulting in cranio-maxillary dissociation.

The Le Fort I fracture extends horizontally across the maxilla above the level of the roots of the teeth, traversing the lower lateral walls of the pyriform aperture and the lower nasal septum. It extends posteriorly across the lateral, medial and posterior walls of the maxillary sinus and involves the pterygoid plates. Unique to Le Fort I fracture is the involvement of the lateral walls of the pyriform aperture.

The Le Fort II fracture is pyramidal, with the apex at the naso-frontal suture. From the apex, the fracture line extends inferolaterally through the medial wall of the orbit, orbital floor, inferior orbital rim and through the zygomatico-maxillary suture. It also extends posteriorly to the pterygoid plates. Unique to Le Fort II fractures is involvement of the inferior orbital rim.

The Le Fort III, also known as cranio-facial disjunction, is like the Le Fort II, and comprises dissociation of the naso-frontal suture. However, this is horizontally oriented, traversing the 
medial and lateral walls of the orbits, the zygomatico-frontal suture and the zygomatic arch. Involvement of the latter is unique to type III fractures ${ }^{2,3}$ (Figure 1).

Some authors have suggested the addition of the Le Fort type $\mathrm{IV}$, which is the propagation of any Le Fort-type fracture to the orbital apex (with a frontal sinus fracture). Identification of involvement of the latter preoperatively is of paramount importance because considerable force is often required to reduce Le Fort fractures with a disimpaction forceps. If the surgeon is unaware, this can pose significant risk of inadvertent injuries to vital structures around the orbital apex, including the internal carotid artery. ${ }^{4}$

Le Fort's classification is today one of the most widely used and understood classifications. In modern clinical practice, however, patients treated at a quaternary-level referral institution, such as Inkosi Albert Luthuli Central Hospital
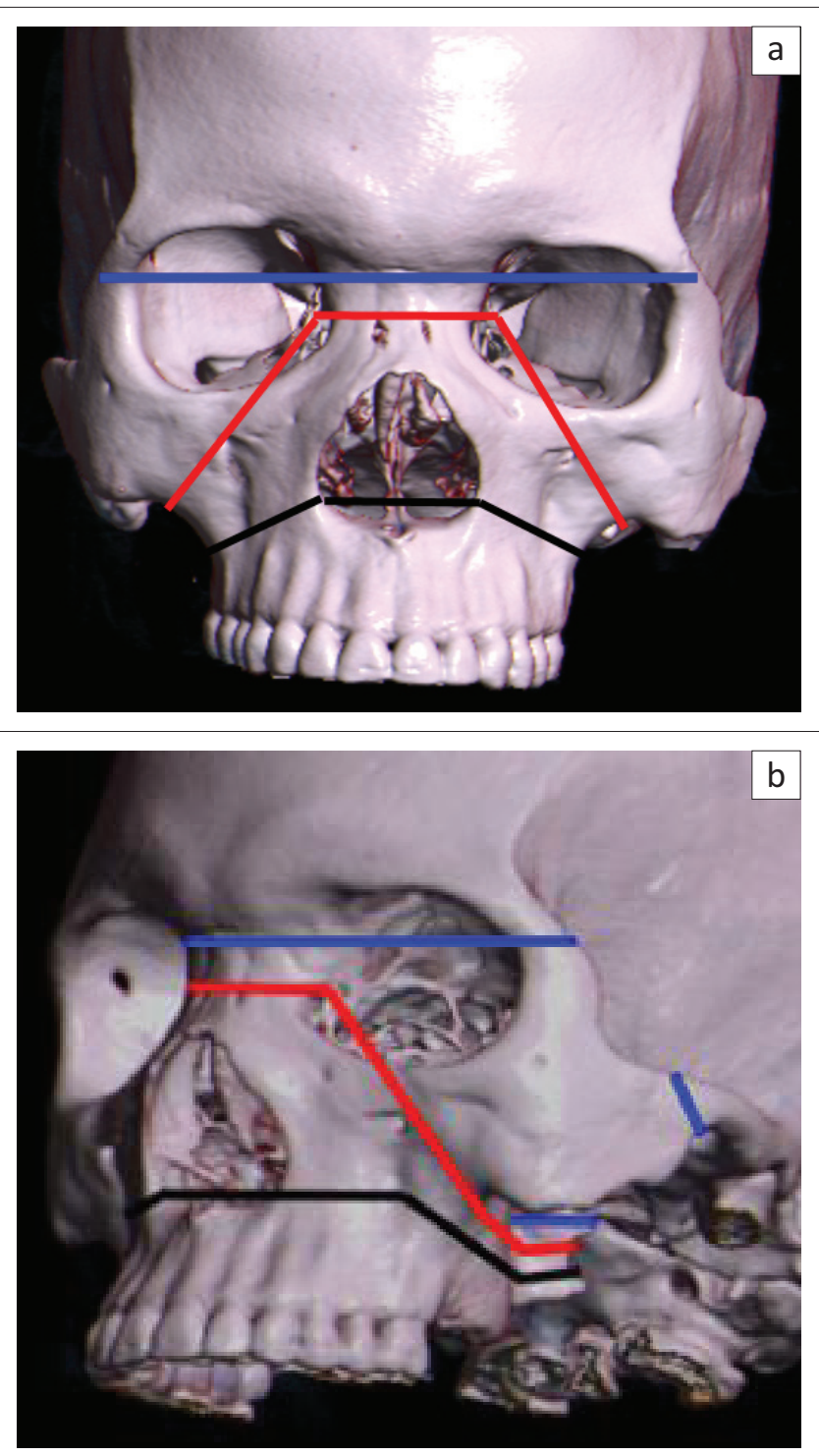

FIGURE 1: Le Fort fracture patterns: (a) AP view of a 3D reformat of a facial bone $\mathrm{CT}$; and (b) left oblique view. Le Fort I (black line), Le Fort II (red line) and Le Fort III (blue line). Note how all Le Fort patterns extend posteriorly to involve the pterygoid plates in (b).
(IALCH), present following mainly high-velocity trauma, which commonly follows motor vehicle collisions. This circumstance is in contrast to Le Fort's work, which was based on low-velocity trauma.

The purpose of the present descriptive observational study was to define facial bone fracture patterns occurring in patients involved in high-velocity blunt trauma, admitted to a quaternary-level referral centre. The traditional Le Fort classification was applied. More importantly, however, the prevalence of variations from the classic Le Fort classification was evaluated, including:

- unilaterality,

- occurrence of multiple Le Fort fractures on one side of the face,

- occurrence of multiple Le Fort fractures at different levels, and on different sides of the face, and

- occurrence of other fractures in addition to Le Fort fractures.

Radiologists' awareness of these different variations is essential for patient care. Accurate diagnosis and classification of facial fractures is crucial in their management, as it assists the surgeon in planning surgical access and the order in which to fix various fractured components. For instance, if a Le Fort I fracture has an associated sagittal or parasagittal fracture of the hard palate extending to the alveolar ridge, this would require fixation to re-establish normal dental occlusion prior to fixation of the maxilla. The primary goals are for the surgeon to restore normal function (such as dental occlusion, sinus clearance, vision and airway patency) and cosmesis (i.e. facial projections and facial height). ${ }^{4}$

\section{Methods}

The study design was of the retrospective observational descriptive type. A pre-existing list of trauma patients with facial bone fractures following high-velocity blunt facial trauma was obtained from the Inkosi Albert Luthuli Trauma Service. It contained the following information: patient age, gender, mechanism of injury and injuries sustained. These data were already present in an approved Biomedical Research Ethics Committee (BREC) Class Approval for these types of studies (BE207-09). IALCH uses a picture archiving and communication system (PACS), enabling patient files and all imaging studies to be readily retrievable from the Hospital and Radiology Information Systems respectively. All cases admitted to IALCH Trauma Service between April 2007 and March 2013 with facial fractures were reviewed. Both male and female patients were evaluated, and of all ages. Children were included because they have similar fracture patterns to those seen in adults, despite their different anatomical and physiological craniofacial skeletal characteristics. ${ }^{5}$

High-velocity trauma included mechanisms such as motor vehicle and motorcycle collisions and severe assault. Although it is practically impossible to quantify the amount 
of force responsible for the facial injuries in each patient, it is generally accepted that in motor vehicle collisions, randomly distributed high-velocity forces are usually involved.5,6,7 In addition, motor vehicle collisions are the most common cause of complex maxillo-facial fractures in developing countries. ${ }^{8}$

The patients had CT scans done with a Siemens Somatom 64-slice multidetector scanner (Siemens, Germany). The following CT scanning protocol was applied:

- Unenhanced scan with a field of view from just above the frontal sinuses to the hyoid bone to include the entire mandible. Axial sections were acquired in a plane parallel to the hard palate.

- Submillimetre slices $(0.625 \mathrm{~mm})$, with overlap $(0.4 \mathrm{~mm})$ obtained for best three-dimensional (3D) volume rendering and multiplanar reconstruction (MPR), using bone and soft tissue algorithms.

- The scans were reviewed at a radiology reporting station: 2 mm slice thickness images; axial, coronal and sagittal multiplanar reconstruction (MPR), with the coronal and sagittal planes perpendicular to the hard palate; and 3D images were reviewed. Chen et al. ${ }^{9}$ and Saigal et al. ${ }^{10}$ reported that whilst $2 \mathrm{D}-\mathrm{CT}$ is useful for tiny and deepstructure fractures, 3D-CT is the best imaging method for the diagnosis of Le Fort-type fractures and can provide valuable information on spacial relationships of fragments, especially for surgical planning.

The scans were read by a junior radiology consultant to define the fractures and classify them using the Le Fort classification. Each case was then classified into categories (Box 1).

Patients whose scans were irretrievable on the PACS, or incomplete on the Radiology Information System (RIS) were excluded. Additionally excluded were patients admitted with imaging from external referring hospitals, as the required reconstructed images were not available. Patients with facial injuries that were detected on radiographs and not evaluated by CT scan were also excluded; these would include isolated nasal bone fractures, which are usually adequately evaluated by radiographs and treated on an outpatient basis. High-velocity facial fractures are usually complex, requiring evaluation with cross-sectional imaging. CT scan is currently the modality of choice for this purpose.

BOX 1: Cases with and without Le Fort fractures.

Cases without Le Fort fractures
Cases with Le Fort fractures
- Classic Le Fort fracture - bilateral symmetrical Le Fort fractures at one level,
with no additional fractures
- Cases with Le Fort plus additional fractures:
bilateral symmetrical Le Fort fractures at one level, with additional
fractures
- bilateral symmetrical Le Fort fractures at more than one level, with no
- bilational fractures
additional fractures Le frical Lort fractures at more than one level with
- Unilateral Le Fort fracture with or without additional fractures:
- several Le Fort fractures on one side of the face, with or without additional
- Bilateral Le Fort fractures with more than one level involvement on at least one
side with or without additional fractures

Facial fractures resulting from penetrating injuries were also excluded from the study.

\section{Results}

A total of 82 cases were retrieved from the IALCH Trauma Service data (BE 209-07). Following the described inclusion and exclusion criteria, the final sample size was reduced to 52 cases. Twenty cases were excluded as there were no records of facial bone CT scans having been performed on the RIS. Four cases could not be retrieved from the archive. One case had no facial bone fractures, the patients in 2 cases died before the scans could be performed (owing to haemodynamic instability) and 3 patients presented with imaging from referring hospitals.

Of the 52 cases, $34(65 \%)$ of the subjects were male and 18 (35\%) were female. Their ages ranged from 2 to 72 years, with a mean age of 30 years. In 50 cases, the mechanism of injury was motor vehicle accident injury. Twenty-four (46\%) subjects were pedestrians, 19 (37\%) were passengers and 7 (13\%) were drivers. Assaults comprised only 2 cases (4\%) of the sample.

In the present study, 12 (23\%) patients had Le Fort fractures, but only 1 had a true Le Fort injury, which was bilateral, symmetrical and on one level. Nine $(75 \%)$ had Le Fort plus other additional fractures. A unilateral Le Fort fracture only occurred in 1 patient, whilst 4 patients had Le Fort injuries bilaterally with more than one level involvement on at least one side. The details are presented in Table 1 and Table 2.

\section{Discussion}

The present study illustrates that at a quaternary level referral hospital, such as IALCH, where most patients present following high-velocity trauma, variations of the traditional Le Fort classification occur more commonly than the classic Le Fort fractures. The latter are commonly depicted in the literature as occurring at one level and symmetrically on both sides of the face.

TABLE 1: Cases of facial bone fractures at IALCH between April 2007 and March 2013.

\begin{tabular}{lc}
\hline Cases & Number of cases \\
\hline Total number of cases & 52 \\
Cases without Le Fort fractures & 40 \\
Cases with Le Fort fractures & 12 \\
$\begin{array}{l}\text { Classic Le Fort fractures - bilateral symmetrical Le Fort } \\
\text { fractures at one level with no additional fractures }\end{array}$ & 1 \\
$\begin{array}{l}\text { Cases with Le Fort plus additional fractures (see Table 2) } \\
\text { Bilateral symmetrical Le Fort fractures at one level, with }\end{array}$ & 9 \\
additional fractures & 1 \\
$\begin{array}{l}\text { Bilateral symmetrical Le Fort fractures at more than one } \\
\text { level with no additional fractures }\end{array}$ & 1 \\
$\begin{array}{l}\text { Bilateral symmetrical Le Fort fractures at more than one } \\
\text { level with additional fractures }\end{array}$ & 1 \\
$\begin{array}{l}\text { Unilateral Le Fort fracture - a single Le Fort fracture on } \\
\text { one side of the face with or without additional fractures }\end{array}$ & 1 \\
$\begin{array}{l}\text { Several Le Fort fractures on one side of the face with or } \\
\text { without additional fractures }\end{array}$ & 3 \\
$\begin{array}{l}\text { Bilateral Le Fort fractures with more than one level } \\
\text { involvement on at least one side with or without } \\
\text { additional fractures }\end{array}$ & 4 \\
\hline
\end{tabular}


In the present study, this classic fracture pattern was found in only one case. This might have been because, in Le Fort's experiments, these injuries were inflicted by blows directed centrally at different levels of the midface, as there was significant difficulty in directing these forces to the lateral aspect of the maxilla, without hitting the zygoma or the mandible. ${ }^{1}$ This consideration may also account for the high association between Le Fort and mandibular fractures, as well as Le Fort and zygomatico-maxillary complex fractures, which were seen in $7(58 \%)$ and $4(33 \%)$ of the cases respectively (Figure 2, Figure 3). In a trauma setting, different forces are applied from endless direction and angle variations. Combined Le Fort and mandibular injuries are thought to be secondary to impact from a broad object to the mandible and the midface simultaneously. The zygoma is the most anteriorly projected bone in the facial skeletal structure, making it prone to injury from any blow to the antero-lateral midface. ${ }^{11}$ This finding matches that of the study by Chen et al. comparing 2D and 3D CT, where $88.7 \%(55 / 62)$ of patients with Le Fort fractures were found to have co-existent fractures in the maxillofacial region. ${ }^{9}$

The total number of Le Fort injuries in the series under discussion was $12(23 \%)$. This figure is comparable to that of $25.5 \%$ found on a much larger study of 663 cases by Turner et al. on patients investigated for possible facial fractures. ${ }^{12}$ Similar to the current study, theirs was also a retrospective study performed at a level 1 referral trauma centre. This relatively low incidence of Le Fort injuries in both studies is thought to be owing to inclusion of cases with isolated mandibular fractures, which contributed to 14 cases $(27 \%)$ in the current study. These fractures result from a direct impact to the mandible without involvement of the midface. In addition, facial bone injuries resulting from the propagation of skull vault and base of skull fractures were also included. These patterns of fracture extensions were seen in 2 cases in the current study, where one was an extension of a frontal bone fracture to the frontal sinuses. The second case was involvement of the greater wing of the sphenoid, which forms the lateral wall of the orbit, and was therefore classified under facial bones. The skull and facial bones are flat bones

TABLE 2: Cases with Le Fort plus additional fractures.

\begin{tabular}{lll}
\hline Patient & Le Fort type & Additional fractures \\
\hline 1 & Bilateral type I and II; left type III & $\begin{array}{l}\text { Body of right mandible; dislocation } \\
\text { left TMJ }\end{array}$ \\
\hline 2 & Left types I, II and III & $\begin{array}{l}\text { Right and left parasymphysial } \\
\text { mandibular fractures; left nasal } \\
\text { bone; hard palate } \\
\text { Left parasymphysial mandibular } \\
\text { fracture }\end{array}$ \\
\hline 3 & Bilateral type I & $\begin{array}{l}\text { Right parasymphysial mandibular } \\
\text { fracture; left ZMC; parasagittal } \\
\text { maxillary fracture }\end{array}$ \\
5 & Bilateral type I; Left type II & $\begin{array}{l}\text { Flail mandible; hard palate; nasal } \\
\text { bone }\end{array}$ \\
\hline 7 & Bilateral type I; left types II and III & $\begin{array}{l}\text { Bilateral mandibular rami NOE; left } \\
\text { ZMC }\end{array}$ \\
\hline 9 & Right types I, II and III & $\begin{array}{l}\text { Coronoid process of right mandible; } \\
\text { left ZMC, NOE } \\
\text { Left ZMC; roof of right mandible }\end{array}$ \\
\hline
\end{tabular}

TMJ, temperomandibular joint; ZMC, zygomatico-maxillary complex; NOE, naso-orbitalethmoidal fractures. that, when fractured, are split by long fissures which are not limited in extension by suture lines.

In Le Fort's experiments, after the infliction of injuries to the facial skeleton, the skull was sawed open and the dura mater stripped off the bone to examine the facial bones for fractures. The specimens were then boiled and macerated to easily remove the flesh. With this process of specimen preparation, by Le Fort's own admission, it was impossible to avoid

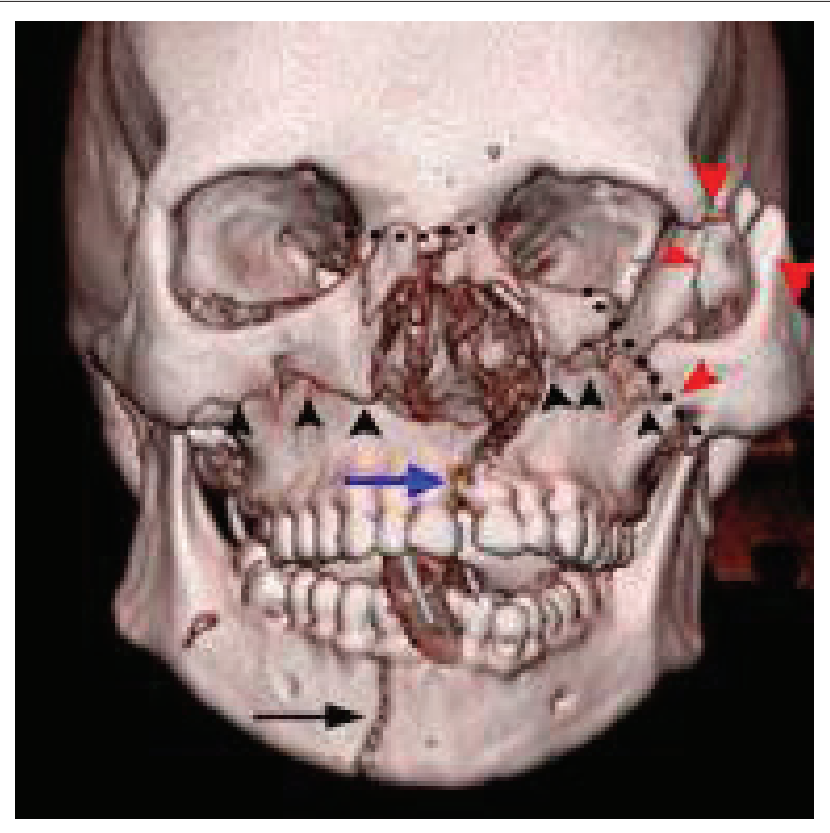

MVC, motor vehicle collision; ZMC, zygomatico-maxillary complex.

FIGURE 2: 3D reconstructed CT scan of the facial bones of a 38-year-old man involved in a MVC as a driver. Bilateral Le Fort I (black arrowheads) and left Le Fort II fractures (dotted lines). Note the presence of the left ZMC (red arrowheads) and right parasymphysial mandibular fracture (black arrow), which are the two most common associations with Le Fort injuries. In addition, there is a sagittal fracture of the maxilla extending to the alveolar ridge (blue arrow).

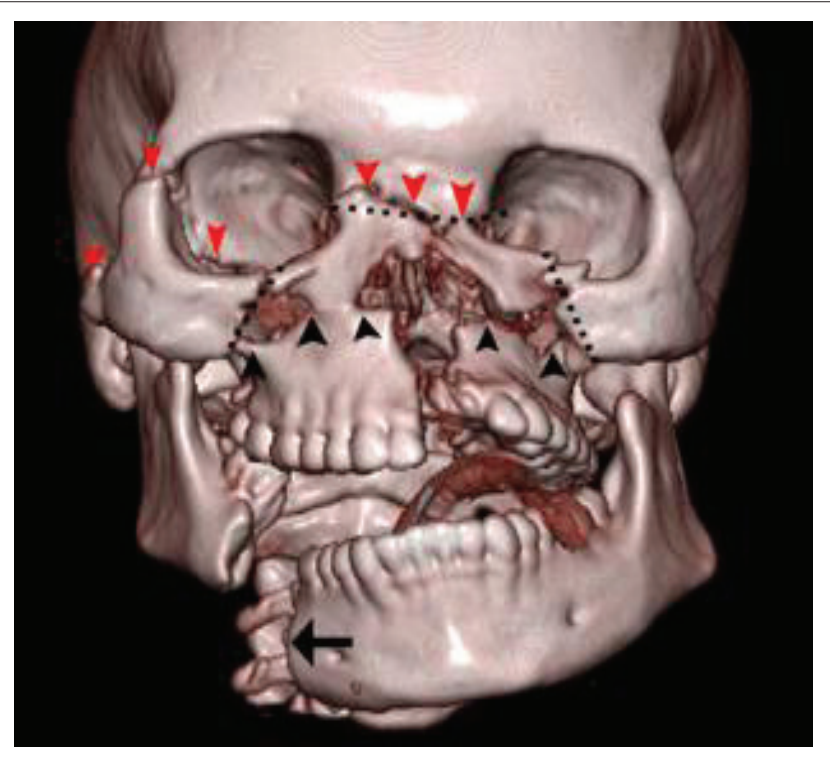

FIGURE 3: 3D reconstructed CT scan of the facial bones of a 40 -year-old woman involved in a MVC as a passenger, showing bilateral markedly displaced Le Fort I (black arrowheads), Le Fort II (dotted line) and right Le Fort III (red arrowheads) fractures. Additionally, there is a fracture of the body of the right mandible (black arrow) and dislocation of the left temporomandibular joint (not shown). 
creating additional fractures. In current practice, however, high-resolution CT scan, which is the standard point of reference, ${ }^{13}$ is readily available in most institutions. With CT scan, fractures can be studied non-invasively. In addition, deep-seated fractures in the very complex facial bone structure can be evaluated with ease; these may otherwise be missed at surface views. Furthermore, CT scan allows rapid acquisition of images in often critically injured patients and adds additional value in assessing the soft tissues (especially intra-orbital structures) and associated brain injuries. Patients with severe facial trauma often have co-existing cervical spinal injuries, and CT scan eliminates the need to manipulate the head into different positions, which is always required in plain radiography.

The mechanism of injury in the current study was overwhelmingly motor vehicle collisions (MVCs), which accounted for $96 \%$ of the cases. Interestingly, this was in contrast to the findings of a large multicentre European study on the epidemiology of facial bone fractures, where MVCs contributed only $11 \%(375 / 3396)$ of the study population and assaults were the leading cause, contributing 39\%. A decreasing trend of MVCs (which used to be the most common cause of facial bone fractures) in developed countries has been documented in recent years, and has been attributed to changes in road traffic legislation. ${ }^{14}$ Stringent traffic policy-making and enforcement are necessary in developing countries to prevent these sometimes devastating injuries.

It is generally accepted to regard motor vehicle and motorcycle collisions as high-velocity trauma, which was clearly established by Luce et al. ${ }^{6}$ as far back as 1979 . It remains, however, practically impossible in live patients to quantify the magnitude of the forces involved, which is one of the limitations of the present study. This shortcoming is in contrast to studies performed in cadaveric skulls where some quantification of the forces involved may be estimated. Notably, on Le Fort's experiments, there is use of words such as 'minimal', 'moderate' or 'violent' with respect to the amount of force exerted to inflict injuries. Furthermore, exact directions and locations of the forces applied could be documented.

Another limitation to the present study was the small sample size, which was owing to unavailable or incomplete data, and is common in retrospective studies. A further prospective study with a larger sample size would be recommended.

Precise recognition of fracture complexes such as Le Fort types, the pattern variants and additional associated fractures is pivotal in their management. Radiologists, if unaware of these different variations, may miss Le Fort fractures and instead create a long list of seemingly isolated fractures, without clinical context or categorisation of fracture severity and criticality. All these variables are important to the surgeon in determining the treatment plan, surgical access, incisions, order in which to fix the different fractured components, expected treatment outcomes and prognostication.
To exclude or include Le Fort fractures in a patient with several facial bone fractures, it is suggested that sequential examination of the facial bones be performed one side at a time, starting at the pterygoid plates and body of the pterygoid bone. The presence of fractures in this area should raise the suspicion of a Le Fort injury as, by definition, all Le Fort fractures involve the pterygoid. Thereafter, fractures unique to a particular Le Fort level should be specifically sought. The easiest place to start is at the zygomatic arches (for Le Fort III), then the inferior orbital rims (for Le Fort II) and then the pyriform aperture (for Le Fort I). Finally, follow a sequential search for the rest of the components of a particular fracture level. The mandible and zygomatic bone fractures (tripod or malar complex injury) should then be sought, as they were found to be common associations with Le Fort injuries.

\section{Conclusion}

Le Fort's classification, based on low-velocity trauma, is one of the most widely used facial bone fracture classification systems. The present study demonstrates that high-velocity facial fractures seen at a quaternary level hospital in modern practice, often deviate from the traditional Le Fort classification. Precise recognition of these variables and additional associated fractures is pivotal in their management. It assists the surgeon in determining the treatment plan, appropriate surgical access and the order in which to fix different fractures. In addition, it allows accurate prognostication of treatment outcomes.

\section{Competing interests}

The authors declare that they have no financial or personal relationships which may have inappropriately influenced them in writing this article.

\section{Authors' contributions}

S.C.M. was the principal investigator and wrote the manuscript. T.H. was the supervisor of the study and made conceptual contributions towards the study as well as manuscript editing.

\section{References}

1. Le Fort. Experimental study of fractures of the upper jaw. Rev chir de Paris 1901; 23:208-227. http://dx.doi.org/10.1148/rg.331125080

2. Winegar B, Murillo $H$, Tantiwongkosi B. Spectrum of critical imaging findings in complex facial skeletal trauma. Radiographics 2013;33:3-19. http://dx.doi. org/10.2214/ajr.184.5.01841700

3. Rhea J, Novelline R. How to simplify the CT diagnosis of Le Fort fractures. Am J of Roentgenol 2005;184:1700-1705.

4. Fraioli R, Branstetter B, Deleyiannis F. Facial fractures: Beyond Le Fort. Otolarygol Clin N Am 2008;41:51-76. http://dx.doi.org/10.1016/j.otc.2007.10.003

5. Alcala-Galliano A, Arribas-Garcia I, Martin-Perez M, et al. Paediatric facial fractures: Children are not just small adults. Radiographics 2008;28:441-461. http://dx.doi.org/10.1148/rg.282075060

6. Luce EA, Tubb TD, Moore AM. Review of 1000 major facial fractures and associated injury. Plast Reconstr Surg 1979;63(1):26 http://dx.doi.org/10.1097/00006534197901000-00005

7. Pappachan B, Alexander M. Biomechanics of craniofacial trauma. J Maxillofac Oral Surg 2012 Jun;11(2):224-230. http://dx.doi.org/10.1007/s12663-011-0289-7

8. Rasmané $B$, Dakouré $P$, et al. Epidermiology of facial fractures: an analysis of 349 patients. Med Buccale Chir Buccale 2014;20:13-16. http://dx.doi.org/10.1051/ $\mathrm{mbcb} / 2013099$ 
9. Chen WJ, Yang YJ, Fang YM, Xu FH, Zhang L, Cao GQ. Identification and classification in Le Fort type fractures by using $2 \mathrm{D}$ and $3 \mathrm{D}$ computed tomography. Chin J Traumatol. 2006;9:59-64.

10. Saigal K, Winokur R, Finden $S$ et al. Use of three-dimensional computerized tomography reconstruction in complex facial trauma. Facial Plastic Surgery 2005; 21(3): 214-219. http://dx.doi.org/10.1055/s-2005-922862

11. Mehta N, Butala P, Bernstein M. The imaging of maxillofacial trauma and its pertinence to surgical intervention. Radiol Clin N Am 2012;50:43-57. http://dx. doi.org/10.1016/j.rcl.2011.08.005
12. Turner GB, Rhea JT, Thrall JH et al. Trends in the use of CT and radiography in the evaluation of facial trauma, 1992-2002. Implications for current costs. Am J Roentgenol 2004;183(3):751-754. http://dx.doi.org/10.2214/ajr.183.3.1830751

13. Hopper R, Salemy S, Sze R. Diagnosis of midface fractures with CT: what the surgeon needs to know. Radiographics 2006;26:783-793. http://dx.doi. org/10.1148/rg.263045710

14. Boffano P, Roccia F, Zavattero E et al. European maxillofacial trauma project: A multicenter and prospective study. Journal of Cranio-Maxillo-Facial Surgery 2015;43:62-70. http://dx.doi.org/10.1148/rg.263045710 